\title{
Les enjeux lexicaux de la lecture littéraire
}

\section{J.-C. Chabanne, M. Cellier, M. Dreyfus, Y. Soulé (IUFM de Montpellier - INRP)}

Deux questions se croisent dans cette contribution : quels problèmes spécifiques pourraient poser aux élèves les usages «littéraires » du lexique? La «lecture littéraire » est elle une activité propice à des apprentissages lexicaux spécifiques?

\section{Le lexique dans le texte littéraire : un fonctionnement spécifique ?}

Même si on se refuse à isoler le texte littéraire de l'ordinaire des usages, on peut toutefois se proposer d'être attentif à quelques phénomènes à quoi on le reconnait :

- un accroissement de la complexité : vocabulaire plus rare, archaïque, ou réactivation d'acceptions inhabituelles, établissant vers l'encyclopédie des relations plus nombreuses, plus précises ; emplois figurés ou complexes comme les métaphores originales, les syllepses...

- un accroissement de la densité, définie comme la richesse des connections sémantiques sollicitées autour des unités : intensification des interrelations en discours, favorisant au sein du texte des relations plus serrées d'un mot à l'autre, par exemple sur le plan sémantique les phénomènes de connotation ou d'inférences complexes, le tissage de champs sémantiques appuyés sur des sèmes afférents, sur le plan phonographique les effets sémantiques des rimes et assonances... Voir comment le texte est structuré de manière tabulaire, multidimensionnelle, par des réseaux lexicaux hiérarchisés ${ }^{1}$.

- un accroissement de l'incertitude sémantique, dans la mesure où le texte littéraire impose au lecteur de faire preuve d'une inventivité nouvelle, qu'il aventure un code toujours inédit, et qu'il s'avance aux marges de l'indicible, qu'il suppose qu'il y a des choses à dire qui sont impossibles à nommer, à expliciter ; que l'effort même de nommer et d'expliciter dénature et détruit $^{2}$.

- un épaississement fonctionnel, dans la mesure où la relation aux textes suppose que les fonctions habituellement dispersés dans les genres du discours se superposent et s'interpénètrent : penser et ressentir, raconter et expliquer, raisonner et émouvoir, abstraire et faire image, etc. Le littéraire inviterait à un travail souterrain du sens, à un sens sous le texte, à une prolifération des sens, à la différence des discours qui visent à l'exhaustivité, à l'univocité, à la stabilité. Ces derniers sont souvent des discours qui privilégient une définition référentielle du sens, alors que le littéraire privilégierait une définition inférentielle et différentielle du sens ${ }^{3}$.

\section{Une pratique spécifique des textes?}

Même si on refuse que le texte littéraire soit défini par des différences formelles, on doit au moins accorder que la pratique littéraire des textes soit irréductible à toute autre. Elle relève d'une herméneutique, qui cherche à éprouver et à ressaisir dans le commentaire ce que le texte littéraire serait seul à rendre possible. Comme la théorie littéraire l'a montré, la lecture littéraire oscillerait entre deux contraires :

\footnotetext{
${ }^{1}$ C'est par exemple le sens du projet de A.J. Greimas et celui de la sémiotique de Paris, depuis Sémantique structurale (1966).

${ }^{2}$ Beaude P.-M., «Ecriture, imaginaire et enjeux symboliques », dans La scolarisation de la littérature de jeunesse, Univ de Metz, 1996.

${ }^{3}$ Rastier F., Sémantique et recherches cognitives, PUF, 1991.
} 
2008 : «Les enjeux lexicaux de la lecture littéraire », dans F. Grossmann et S.Plane (éds), Les Apprentissages lexicaux : Lexique et production verbale, Villeneuve d'Ascq : Presses du Septentrion. Actes des journées INRP/Lidilem, «Lexique et production verbale : vers une meilleure intégration des apprentissages lexicaux », mars 2005, INRP, Lyon

- d'une part, elle doit s'ouvrir aux possibles du texte, faire proliférer le sens, déclarant ainsi l'œuvre ouverte ${ }^{4}$;

- d'autre part, le texte impose ses propres contraintes et donne d'indispensables limites à l'interprétation $^{5}$ : il y a des interprétations plus aventurées que d'autres. Le risque de dérive interprétative est encore plus sensible avec des lecteurs jeunes, dont l'encyclopédie... et le lexique sont en cours de construction.

Ces limites dépendent d'abord des contraintes du texte lui-même, qui demandent au lecteur des méthodes de lecture même sommaires, appuyées sur des indices textuels, des relevés, des mises en relation, qui se croisent et se confirment. Elles dépendent ensuite de la langue ellemême, définie comme ce qui est censé partagé par les locuteurs. Il n'y a pas de lecture interprétative recevable sans ce qu'on pourrait appeler une lecture philologique ${ }^{6}$, pour la distinguer de ce qui serait une lecture flottante du texte, subjective et aventurée. Enseigner la littérature, c'est justement enseigner les règles de l'interprétation, mêmes sommaires, et apprendre à asseoir sur le texte et la langue une lecture informée, appuyée sur une observation scrupuleuse des indices linguistiques.

Ce travail sur le texte relève de pratiques sociales qui, pour une part, sont spontanées, et qui, pour la part qui nous intéresse, sont des objets d'apprentissage prenant une place importante, en volume, dans les curricula scolaires. Nous nous proposons d'approcher quelques-uns de ces savoir-faire.

\section{Ce que révèlent les difficultés autour des textes}

Une des entrées possibles est d'observer comment les formes de travail sur le lexique, dans la classe de littérature, inhibe ou favorise le travail interprétatif. Une des difficultés classique dans la séance de lecture est le «défrichage lexical » : le repérage des «mots difficiles » et leur éclaircissement, par exemple en cherchant dans le dictionnaire. C'est souvent une impasse : le texte y éclate en un kaléidoscope de difficultés imprévues ; sa logique d'ensemble se dissout dans cet effort d'éclaircissements ponctuels qui devient une impasse.

D'abord parce que la collecte des «mots difficiles » est sans fin, que les difficultés des uns ne sont pas celles des autres. Le texte éclate en une liste informe de vocables, alors même que le dictionnaire, bien souvent, fournit des définitions encore plus abstraites et illisibles, quand il ne propose pas un flot d'acceptions entre lesquelles il est impossible de choisir... sans avoir compris, au moins sommairement, le texte lui-même. Le dictionnaire est lui aussi un texte à interpréter...

En outre, un mot «difficile » dans un texte est rarement un mot technique, archaïque ou peu fréquent. Les mots les plus simples y sont retravaillés par les figures et les ramener à une définition générale ne rend pas compte de leur valeur en contexte. D'où des malentendus lexicaux d'autant plus pernicieux qu'ils sont «invisibles »: souvent ce sont les mots les plus familiers en apparence qui sont les plus déroutants.

Enfin, il faut observer de près les processus d'entrée dans le sens. La compréhension du texte ne se fait pas exclusivement à partir d'une compréhension lexicale fine intégrale (tous les mots seraient connus et leur valeur en contexte établie un par un). On passe le plus souvent par des états intermédiaires d'appréhension du sens, qui font alterner des saisies globales de l'organisation sémantique d'ensemble, et des arrêts sur tel ou tel détail qui impose un recours au sens lexical fin de tel ou tel vocable. Puis on revient à la configuration globale (un résumé,

\footnotetext{
${ }^{4} \mathrm{U}$. Eco, L'Euvre ouverte, Seuil, 1965.

${ }^{5}$ U. Eco, Les Limites de l'interprétation, Grasset, 1992.

${ }^{6}$ M. Burgos parle de « compétence philologique », dans « Lecteurs experts, lecteurs convers », Pratiques 76, déc. 1992 , p. 65 .
} 
2008 : «Les enjeux lexicaux de la lecture littéraire », dans F. Grossmann et S.Plane (éds), Les Apprentissages lexicaux : Lexique et production verbale, Villeneuve d'Ascq : Presses du Septentrion. Actes des journées INRP/Lidilem, «Lexique et production verbale : vers une meilleure intégration des apprentissages lexicaux », mars 2005, INRP, Lyon

un schéma actanciel, une évaluation, etc.) et un autre cycle interprétatif recommence. L'observation des classes montre que l'affinement de la compréhension est parallèle au développement de l'interprétation, et non préalable. Si les élèves ont besoin du sens des mots, c'est d'abord qu'il n'en ont pas tenu compte, mais que le besoin s'en fait sentir à partir du moment où le débat montre des désaccords.

Entrer dans le texte par une saisie globale peu à peu affinée permet aussi de résoudre le choix des mots sur lesquels on va porter l'attention. On observe que très vite les premières interprétations vont donner du relief à un nombre réduit de vocables ou d'expressions, qui se révèlent des mots-pivots, des mots-clés, carrefours de l'interprétation: mots-clés pour les lecteurs, les mots-clés de leur lecture, mais qui vont souvent retrouver ceux qu'une lecture experte décèlerait. Le travail lexical fin peut alors se faire sur des éléments pertinents et cohérents avec la lecture d'ensemble.

Une telle démarche peut être rapprochée des processus d'auto-apprentissage du lexique. On apprend le lexique non en combinant des unités sémantiques pour comprendre les énoncés, mais au contraire en les dégageant progressivement de leurs combinaisons, d'une part en tenant compte du contexte et de la situation, d'autre part, en faisant appel à son dictionnaire personnel, qui s'enrichit, se structure et s'affine ainsi peu à peu. Le travail proprement métalinguistique, qui consiste à échanger par exemple sur la morphologie, la définition, la distinction entre paronymes, etc. intervient en cas de nécessité ${ }^{7}$.

Ce phénomène n'est pas différent que ce lui qu'on observe dans l'évolution lexicale. Les usages précèdent toujours l'enregistrement dans la norme (la lexicalisation). Ce n'est que progressivement qu'un vocable voit sa nébuleuse sémantique se décanter, se condenser, se stabiliser, et la définition du dictionnaire n'est en fait qu'une saisie synchronique (toujours provisoire) de ce mouvement de condensation et de stabilisation du sémème.

Ce processus d'apprentissage qui va à l'encontre des conceptions cumulatives du lexique est bien décrit par les développementalistes (voir les contributions de K. Duvignau et E. Nonnon). Pourquoi ne serait-il pas à l'œuvre quand il s'agit d'acquérir les compétences nécessaires en lecture littéraire ? À une conception positiviste stricte, qui postule qu'un texte doit se comprendre d'abord, peut-être faut-il substituer une représentation plus complexe et plus circulaire de l'acquisition: en situation de lecture littéraire, un texte s'éprouve sensuellement et affectivement, il s'interprète par des paraphrases globales ; la compréhension dans le détail se fait d'abord de manière très lacunaire, par étapes progressives, et ce n'est que quand le questionnement se fait plus exigeant, que le lecteur s'impose de dégager dans le détail le sens de tel ou tel constituant. C'est justement cette exigence qui caractérise la lecture scolaire.

\section{Comment la littérature enseigne le lexique}

On retrouve alors le problème abordé dans l'ensemble de cet ouvrage : une lecture littéraire des textes sollicite une connaissance fine du lexique, mais alors comment celle-ci est-elle acquise ? Pour une lecture littéraire qui équilibre droit du lecteur et droit du texte ${ }^{8}$, il faut un enseignement du lexique. Mais il serait sans doute contestable de penser que cet enseignement devrait nécessairement précéder le travail de lecture. L'observation des pratiques réelles montre que c'est justement parce qu'ils sont face à des textes qui imposent des modalités de

\footnotetext{
${ }^{7}$ David J., "Le lexique et son acquisition : aspects cognitifs et linguistiques », Le Français aujourd'hui 131 :

"Construire les compétences lexicales", sept. 2000, p. 31-41.

${ }^{8}$ Tauveron C., Lire la littérature à l'école, Hatier, 2002.
} 
2008 : «Les enjeux lexicaux de la lecture littéraire », dans F. Grossmann et S.Plane (éds), Les Apprentissages lexicaux : Lexique et production verbale, Villeneuve d'Ascq : Presses du Septentrion. Actes des journées INRP/Lidilem, «Lexique et production verbale : vers une meilleure intégration des apprentissages lexicaux », mars 2005, INRP, Lyon

lecture spécifiques que les élèves vont apprendre du vocabulaire et la particularité de ses usages dans ces drôles de textes.

C'est ainsi que le texte littéraire pourrait enseigner le vocabulaire d'une manière tout à fait spécifique, en offrant pour les mots une fiction qui vaudrait définition au sens de la théorie du

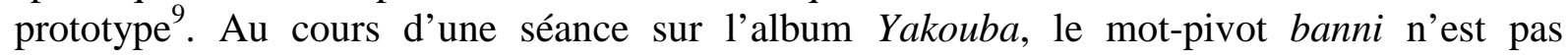
vraiment compris des élèves, qui devinent seulement que c'est un mot clef.

\begin{tabular}{|ll}
\hline M & alors il est banni, il est mis à l'écart du village à garder le troupeau \\
é1 & pour une bonne raison \\
é2 & msieur ça veut dire quoi banni \\
é3 & banni ça veut dire mis à l'écart \\
é4 & banni ça veut dire [inaudible - ton interrogatif] \\
M & non / banni ça veut dire que tu es mis à l'écart / que tu n'es/ \\
é4 & que tu es avec les autres mais que/ \\
M & que tu n'es pas avec les autres justement que tu es tout seul \\
é4 & c'est pour ça qu'il veut pas dire qu'il a pas tué le lion
\end{tabular}

Ils se montrent tout à fait capables d'associer ce mot à la fiction elle-même, qui sert désormais de référence, à la manière des frames et scripts des psycholinguistes : "être banni, c'est comme Yakouba ». On est encore loin de la définition analytique par l'énumération des sèmes ou par le renvoi à un référent. Le fragment de récit tient lieu d'exemple, et pas de manière sommaire : il comporte ses nuances et ses ambiguïtés. Ce fragment narratif permet de cerner subtilement le sens de la notion avant d'en venir au discours explicatif. On peut penser qu'il s'agit d'une pré-compréhension, encore très flottante, non paraphrasable; mais le savoir lexical se construira sur cette expérience initiale.

Et si ce qu'offrait la littérature à l'enseignement du lexique, c'était cette dimension proprement «expérientielle »: la pratique littéraire du texte consiste justement à «vivre le sens » en vivant physiquement et affectivement le texte ? C'est en ce sens que l'interprétation pourrait nourrir la compréhension : c'est la construction d'un univers fictionnel riche et complexe qui va informer les mots du texte, à la manière d'une vaste allégorie. On n'est pas encore dans un usage explicite de la définition - qui suppose un lourd travail encyclopédique - mais dans l'association d'un fragment fictionnel complexe et d'un vocable. Peut-on aller jusqu'à dire que l'œuvre littéraire fournit pour la mémoire du lecteur ce qui est l'équivalent des exemples du dictionnaire : un contexte prototypique, une micro-fiction, qui permet de « visualiser/imaginer » le sens, de le «sentir » intuitivement.

Rattacher un vocable à des (con)textes précis, appelés dans toute leur complexité à constituer sa valeur et sa définition, peut-être n'est-ce là pas autre chose qu'une définition de la culture. Au dictionnaire et à l'encyclopédie, il faudra penser à ajouter la bibliothèque à la liste des outils de la lexicologie.

Deux autres expérimentations peuvent étayer cette analyse.

\section{Entrer dans un texte par les mots.}

La question que le texte adresse au lecteur ne se trouve pas dans un des constituants ni dans la mise bout à bout de ces derniers. La compréhension réelle d'un texte et son interprétation ne peuvent naître que de l'ensemble qui transcende les phrases et mots. Mais ceux-ci restent porteurs de sens : le rapport herméneutique se joue entre le littéral des mots et le figuré du

\footnotetext{
${ }^{9}$ G. Kleiber, Sémantique du prototype, PUF, 1990.
} 
2008 : «Les enjeux lexicaux de la lecture littéraire », dans F. Grossmann et S.Plane (éds), Les Apprentissages lexicaux : Lexique et production verbale, Villeneuve d'Ascq : Presses du Septentrion. Actes des journées INRP/Lidilem, «Lexique et production verbale : vers une meilleure intégration des apprentissages lexicaux », mars 2005, INRP, Lyon

texte pour se déployer au fur et à mesure en plusieurs phases «interprétatives » jusqu'à la stabilisation définitive du sens.

Dans une de nos expérimentations, nous avons voulu contraindre ce va-et-vient entre la globalité du texte et les mots-pivots et donner la primauté à ces derniers : comment les élèves s'appuient-ils sur eux pour progresser? Comment reviennent-ils à eux, comme étapes décisives, après le continuum de la lecture ? Quelles sont les procédures mises en œuvre pour les comprendre, les intégrer, les ignorer ou en contourner l'opacité ? Le dispositif permet d'entrer de façon plus précise dans la manière dont les enfants appréhendent l'unité-mot et pas seulement le mot difficile qui n'est qu'une catégorie parmi d'autres, la plus contestable, d'ailleurs. Le travail a été effectué par une PEMF, Soisic Bozec, dans un CM1 nîmois, situé en ZEP, à partir d'un texte dit « littéraire »- même si on ne veut pas entrer dans le détail de ce qu'est la littérarité, on peut légitimer ce caractère ici par la figure de l'auteur, Anton Tchekhov. «Le miroir déformant» est une nouvelle subtile, de tonalité fantastique :

Un homme entraîne son épouse dans la demeure de ses ancêtres très poussiéreuse et aux murs verdis par le temps. Il lui parle de sa bisaïeule qui, d'après la famille, s'était toquée d'un miroir aux propriétés magiques; elle ne s'en séparait jamais et aurait même voulu l'emporter avec elle dans son cercueil. L'homme secoue la poussière et découvre en riant qu'il s'agit d'un miroir déformant. Sa femme s'approche, se regarde, pousse un grand cri et s'évanouit. Revenue à elle, elle ne cesse de le réclamer et reproduit le scénario de la bisaïeule: elle s'y regarde jour et nuit. Dix ans après, par hasard, l'homme découvre le secret: le miroir déforme en effet mais les traits de sa femme, fort laids et irréguliers, sont déplacés de telle façon qu'elle devient extrêmement belle. Le phénomène le fascine tellement qu'il reste des heures à contempler sa femme dans ce fameux miroir.

Le dispositif est le suivant :

Séance $1: 1^{\text {ère }}$ lecture du texte par l'enseignante. Consigne donnée après la lecture : chaque enfant va lui dire à l'oreille le mot de cette histoire qu'il a envie de garder. Tableau de ces mots : 3 sur les personnages (l'homme, la femme, la grand-mère), 4 sur les valeurs positives (Amour, heureux, si belle, que je suis belle), 4 sur la déformation (déformé, figure déformée, nez tordu, menton coupé), 2 sur la mort ("quand elle tombe dans le coma », " un mot qui veut dire cercueil que j'ai oublié » - la bière) 7 fois miroir et 1 fois miroir déformant.

Séance $2: 2^{\text {ème }}$ lecture du texte : Consignes : noter dans un tableau en 3 colonnes «les mots importants », « les mots qui plaisent », « les mots incompris ». Un élève propose de noter au dos du tableau ceux « qu'on n'aime pas ». Mise en commun des mots sur un tableau collectif en 4 colonnes.

Séance 3: $\quad 3^{\text {ème }}$ lecture par l'enseignante ; les élèves ont le texte sous les yeux. Les listes de mots choisis en séance 2 sont affichés au tableau; chaque enfant doit choisir un mot dans chaque catégorie et justifier ses choix individuellement. Dans la liste des mots incompris, chacun repère ceux qu'il comprend et les explique par écrit.

Séance 4: restitution du récit pour vérifier les interprétations. A part 4 réponses très éloignées du texte, 3 solutions sont avancées : 10 élèves disent que le miroir déforme ; 4 pensent qu'il y a une dame dans le miroir, 4 autres affectent au miroir un pouvoir magique : à peine plus de la moitié de la classe donne le sens du texte porté par le titre : «le miroir déformant $\gg$.

Séance 5: On revient sur les réponses données à la séance 4. L'enseignante relit le texte et demande aux élèves, en fonction de la solution choisie, de surligner les éléments la justifiant : le réseau lexical renvoie à l'interprétation. Après quoi, a lieu une discussion collective autour des deux interprétations importantes : explication physique (miroir déformant) vs explication fantastique (miroir doté d'un pouvoir magique), ce qui débouche sur un petit 
2008 : «Les enjeux lexicaux de la lecture littéraire », dans F. Grossmann et S.Plane (éds), Les Apprentissages lexicaux : Lexique et production verbale, Villeneuve d'Ascq : Presses du Septentrion. Actes des journées INRP/Lidilem, «Lexique et production verbale : vers une meilleure intégration des apprentissages lexicaux », mars 2005, INRP, Lyon

raisonnement philosophique suscité par les élèves eux-mêmes autour des mots «beauté intérieure, beauté extérieure, laideur intérieure, laideur extérieure ».

Séance 6 : On reprend la première liste des mots incompris. Les élèves éliminent ceux qu'ils ont saisis, et définissent les autres. Les mots qui ont été très « discutés » ( ensorceleur - digne de - inhumain - murs verdis - bronze noirci - elle blêmit ) vont faire l'objet d'un mini-récit écrit par les élèves.

L'analyse de l'ensemble du dispositif appelle un certain nombre de remarques rapides :

L'étonnante dispersion des mots et expressions. Au lieu de la concentration attendue autour de quelques mots, on constate un éclatement important, chaque élève ayant retenu deux ou trois unités personnelles, pour chaque rubrique.

La notion de mot a souvent été dépassée, les enfants relevant fréquemment un GN, voire un segment de phrase, sur des critères sémantiques. Par exemple, dans les «mots importants », Yacine a choisi «donne-moi le miroir» «parce que la femme de l'homme elle va mourir si elle ne regarde pas nuit et jour son miroir. » Autrement dit, ce n'est pas l'objet central qui l'a intéressé mais le rapport de la femme avec le miroir : il a visé le manque vital que le mot «miroir » seul ne pouvait traduire.

La structure «nom + adjectif » a eu beaucoup de succès et certains groupes ont été débattus jusqu'au bout (bronze noirci, murs verdis, terrible secret, passion folle, rire inhumain). Les élèves ont été sensibles à l'infléchissement du sens général par l'adjectif et particulièrement par un adjectif peu usuel (verdis/noircis vs vert/noir). Ils ont perçu que l'association ajoute des valeurs sémantiques secondes, du flou, ouvre une perspective : l'adjectif remotive le substantif en lui donnant une autre résonance, d'autres potentialités.

L'interprétation du texte autour des mots pivots : la notion de «mots importants » a paru difficile dans un premier temps : importants pour qui et de quel point de vue, se sont demandé les élèves ? Ce que visait l'enseignante, c'étaient les mots nucléaires autour desquels se construit de manière évidente le sens, comme le terme «miroir» qui a été mentionné effectivement par la majorité de la classe. Si certains se sont ensuite égarés sur des détails annexes (causer, papiers entassés, rats), la plupart - et particulièrement les «bons élèves », de façon attendue - sont restés sur les mots permettant de fixer l'interprétation du texte qui pouvait basculer ou vers une hypothèse réaliste - portée par le titre «le miroir déformant » et le réseau lexical afférant cette idée - ou l'hypothèse fantastique autour d'autres termes pivots. Mais le titre est particulièrement important puisque, signifiant le texte de façon abrégée, il contraint le parcours interprétatif vers la première hypothèse, tout comme le fait à la fin de la nouvelle un paragraphe explicite : «Pourquoi ma femme laide et sans grâce paraissait-elle si belle dans le miroir ? Tout simplement parce que... »

Mais la moitié de la classe environ a passé outre ce verrouillage du sens et a validé l'hypothèse d'un miroir magique. Pourquoi ? Le référent était, certes, peu clair : quelques enfants n'avaient jamais vu ce type de miroir. Ils ne le concevaient pas comme un processus physique parfaitement explicable - la compréhension peut avoir bloqué l'interprétation. Au contraire, le terme «magique » associé à « ensorceleur » est revêtu de connotations fortes qui renvoient à un univers connu de tous, celui du conte et de la métamorphose - le laideron qui devient beau, l'animal qui devient prince. Mais l'élément déterminant est sans doute ailleurs : il ne faut pas négliger la place des mots dans un texte: sa clôture est toujours décisive, particulièrement dans le genre fantastique. Celui-ci n'a pas failli à la règle : la fin est ouverte, relançant l'hypothèse fantastique qui semblait avoir été éliminée, avec de multiples 
2008 : «Les enjeux lexicaux de la lecture littéraire », dans F. Grossmann et S.Plane (éds), Les Apprentissages lexicaux : Lexique et production verbale, Villeneuve d'Ascq : Presses du Septentrion. Actes des journées INRP/Lidilem, «Lexique et production verbale : vers une meilleure intégration des apprentissages lexicaux », mars 2005, INRP, Lyon

expressions qui, à cette place stratégique, ont capturé les petits lecteurs. Les relevés de mots « importants » se situent, pour les tenants de cette hypothèse, dans cette zone.

Les mots qui plaisent : cette rubrique un peu étonnante pouvait permettre de saisir le rapport affectif au texte. Les mots ont généralement été relevés pour le référent, ce qui explique l'abondance de termes autour du champ de la beauté - Nabil a choisi « assis tous les deux devant le miroir » «parce qu'ils sont heureux ensemble »; Zaccharia : «grande beauté » car «c'est un mot qui fait plaisir». D'autres ont été sélectionnés pour l'enveloppe sonore, le signifiant : chandelier, par exemple, a été incompris, écrit «chant de lié » mais considéré comme beau; de même bisaïeule («je l'aime pour ce qu'on entend », dit Alicia ) ou «mon mari ment », répété avec une évidente satisfaction par une élève plusieurs fois.

Certaines expressions, très convenues, quasiment des collocations (merveilleuse beauté, terrible secret, passion folle) étaient neuves pour eux et leur procuraient du plaisir. Plaisir est le terme approprié car c'est ainsi que le texte séduit le lecteur, en tissant un écheveau de mots, d'expressions qui développent un réseau de croyances et de représentations captivantes. Il n'est pas indifférent non plus que les enfants aient relevé des termes créateurs d'atmosphère : lumière, bougie, chandelier. Cette rubrique qui pouvait paraître contestable s'est avérée intéressante parce qu'elle touche à l'univers que suscite la fiction, aux mots qui la créent, et à l'énigmatique rapport des mots et des choses.

Les mots qui ne plaisent pas : on y trouve des termes en rapport avec des notions négatives : la laideur, qui était avec la beauté une des deux grandes isotopies du texte mais aussi la mort ( Massabia rejette mort «parce que c'est un mot de violence») ou des termes en rapport avec les valeurs : « un mot qui me plaît pas c'est ment parce que c'est mal de mentir », dit Phendryl. On peut constater aussi que six expressions présentes dans cette rubrique se trouvent aussi dans la précédente : ma femme est laide, s'arracher les cheveux, rire, ment, bière, tordu. Ce qui fait peur aux uns intéresse les autres, l'adhésion au texte ne se faisant pas sur la même base. Autres remarques : les élèves tiennent compte de l'environnement lexical : «éclat de rire » et « rire inhumain » ne sont pas placés dans la même colonne. Mais quelques uns, très rares, se retrouvent dans les quatre rubriques comme assez curieusement le mot bière («le miroir n'entrait pas dans la bière »). Son caractère polysémique est relevé et à ce titre, il est doublement condamné : «C'est un cercueil », dit Myriam, «mais quelquefois on appelle aussi ce qu'on boit et j'aime pas ». L'aspect culturel n'est jamais négligeable.

Les mots incompris : c'est la rubrique la plus «scolaire», celle où le palier du mot est apparu avec le plus de netteté - deux expressions seulement. Les relevés et explicitations orales collectives ont fait apparaître les zones de flou et l'hétérogénéité des connaissances lexicales de la classe, le même mot pouvant être inconnu, mal connu ou partie du vocabulaire actif de certains.

On y trouve les mots non identifiés, mal segmentés ou mal retranscrits, après les deux lectures magistrales. Puis des termes dont le sens a été vite réglé, comme bisaïeule, qui, monosémique et glosé par le texte a été utilisé à bon escient par les enfants; d'autres dont les emplois se sont révélés problématiques à cause du parasitage de termes proches comme effroyable contaminé par effrayé ( Abdoul : le garçon est effroyable de voler). En revanche, la plupart des termes déclarés «durs » par certains ont été intuitivement plus ou moins compris en contexte, comme inanition ou blêmir ; les élèves se demandaient si l'héroïne devenait rouge, bleue, ou blanche mais ils avaient compris qu'il s'agissait d'une manifestation de l'émotion. Autrement dit, ces termes ont occupé, en gros, la place sémantique qui était la leur. D'une manière générale, leur méconnaissance ou l'infléchissement du sens éventuel n'a pas bloqué 
2008 : «Les enjeux lexicaux de la lecture littéraire », dans F. Grossmann et S.Plane (éds), Les Apprentissages lexicaux : Lexique et production verbale, Villeneuve d'Ascq : Presses du Septentrion. Actes des journées INRP/Lidilem, «Lexique et production verbale : vers une meilleure intégration des apprentissages lexicaux », mars 2005, INRP, Lyon

la compréhension de l'ensemble. Il n'aurait sans doute pas été productif de faire des explications détaillées qui auraient arrêté la traversée du texte. Mais les explicitations autour des autres rubriques ont révélé des méconnaissances beaucoup plus insidieuses que celles affichées ici : les conduites de définition ont montré que des mots simples, en apparence connus, sont en fait mal saisis. Ainsi le mot sévère a été placé par une élève dans les mots qu'elle n'aimait pas car «ça veut dire qu'on est méchante et après on reste toujours méchante ». Elle ne l'a pas identifié comme un mot difficile mais elle en faussait le sens.

Ce dispositif expérimental - esquissé à grands traits - a plu aux élèves, malgré son côté assez contraignant. Il les a obligés à aller et venir des mots au texte, à passer du sens global à l'analyse des effets ancrés dans un seul terme ou expression et pourtant dépendants de contextes plus ou moins lointains et de leur place même dans la nouvelle. Mais il souligne surtout que le rapport au texte de chacun est différent; ce ne sont pas toujours à partir des mêmes éléments que le sens se construit ou se dérobe.

\section{Activités lexicales et interprétation}

Le travail du lexique en littérature permet-il de dépasser le clivage théorique opposant un enseignement en langue et un enseignement en discours d'une part, un enseignement incident et un enseignement systématique de l'autre. Les séances analysées concernent trois classes de CE2 (Annick Berrini, Florence Ozil et Sylvie Waleck) qui ont lu Voyage au pays des arbres de Le Clézio (Gallimard, col. «Folio Cadet», 2003). Ce court récit est l'histoire d'un jeune garçon qui découvre la vie secrète des arbres : l'expérience, avant tout perceptive, sensorielle, se double d'une expérience proprement littéraire : apparemment immobiles et silencieux, les arbres se révèlent pour qui sait voir et écouter particulièrement animés : ils bougent, font du bruit, parlent. Le personnage apprend à communiquer avec eux et à découvrir leur univers. Ce faisant, le lecteur est confronté à une poétique attachée à la transcription des impressions, des sensations, marque de fabrique de l'écriture de Le Clézio. L'auteur exploite les ressources de la reformulation et de la synonymie en une organisation textuelle qui procède par ajouts et reprises. Peu de mots a priori inconnus des élèves et des champs lexicaux nettement repérables. Mais si le propre du texte littéraire est de donner à voir en dernière instance le travail sur la langue, le récit de Le Clézio est particulièrement significatif sur ce point : le lexique du mouvement et du bruit y constitue la matière poétique et sémantique appelant un travail d'analyse (distinction «sourd »/《 sonore » ou «siffler »/»siffloter ») à la mesure de l'enjeu interprétatif : adhésion ou croyance à la vie secrète, anthropomorphique des arbres («Si tu ne fais pas attention, tu peux croire que ce sont les oiseaux qui sifflent. Il faut dire que ça y ressemble beaucoup. Mais ce ne sont pas les oiseaux qui sifflent, ce sont les arbres» p.24).

Nous avons donc élaboré nos séances autour des particularités du lexique du texte afin d'explorer des modalités de compréhension et d'interprétation qui profitent de l'articulation entre des relevés de termes plus ou moins systématiques, des analyse de type «métalexical » et des activités de mime portant sur des expressions-clé.

Caractéristiques du dispositif : il repose sur l'alternance entre des temps de travail individuel et collectif, des phases d'écrits et d'oraux «réactifs » et réflexifs : notation d'impressions sur le carnet de lecture, échanges autour de mots relevés et étudiés, relectures du texte. Il cherche à favoriser un protocole de lecture à la fois singulier et partagé, qui sollicite et accueille les différents niveaux de traitement (inférentiel, référentiel, différentiel), et autorise le retour au texte.

Séance 1 : après un premier contact avec le livre, que les enfants manipulent et feuillettent librement, la consigne donnée est : "je vais vous demander d'ouvrir le livre, de passer la 
2008 : «Les enjeux lexicaux de la lecture littéraire », dans F. Grossmann et S.Plane (éds), Les Apprentissages lexicaux : Lexique et production verbale, Villeneuve d'Ascq : Presses du Septentrion. Actes des journées INRP/Lidilem, «Lexique et production verbale : vers une meilleure intégration des apprentissages lexicaux », mars 2005, INRP, Lyon

page de titre, d'aller à la page 5, uniquement à la page 5 ». S'ensuit une lecture orale par un élève puis l'enseignante note au tableau une phrase du livre "C'est comme ça qu'il a eu l'idée d'aller au pays des arbres. » Elle demande aux élèves d'imaginer la suite de l'histoire. Les hypothèses sont notées sur une affiche.

Séance 2 : Confrontation des hypothèses avec les premières impressions qui suivent la lecture intégrale et individuelle de l'ouvrage.

Séance 3 : Mise en place du carnet de lecture : «Ce carnet de lecture va être à vous, il vous servira de mémoire » Discussion à propos de la fonction du carnet de lecture et de ce qui va être noté dans le carnet : "Que peut-on écrire ou noter dans le carnet de lecture?»

Propositions des élèves : relever et noter «les mots qui m'ont expliqué le texte» ou «les mots ou les expressions qui me font rêver » «les mots ou les expressions que j'ai aimés, qui m'ont plu », « les mots du texte que je retiens », «ce que j'imagine », «ce que j'apprends », «ce que je remarque», «les mots et les moments d'émotion ...». Travail personnel d'écriture.

Séance 4: "Quels sont les mots du texte que vous avez notés dans votre carnet de lecture ? lecture ». Les mots et sont lus, discutés, éventuellement classés, puis inscrits sur une affiche. Explication en contexte des mots incompris par retour au texte (à propos de «taciturne »: «Il faut essayer de comprendre ce mot en regardant dans le texte ce qui l'entoure ». Les élèves prennent alors appui sur le procédé de reprise-reformulation dans l'ouvrage : "Eux ne disent pas grand chose. Ils sont un peu taciturnes,... » (p. 20). Ils apprennent à repérer ce procédé pour comprendre d'autres mots du texte, par exemple «jaser»: «...ils commencent à jaser, ils se racontent des histoires,( ...) sans queue ni tête. Ils parlent de la pluie et du beau temps(...) parlent tout le temps sans s'arrêter » ( p. 22).

Séance 5 : Travail à partir de la consigne : «Soulignez les mots qui font comprendre que les arbres sont immobiles et classez les mots selon un plus ou moins grand degré d'immobilité. Des variantes sont proposées dans les deux autres classes : «Relevez les mots et les expressions qui traduisent le mouvement et le bruit que font les arbres » ou « Recherchez les mots et les expressions qui montrent que les arbres ne sont pas silencieux ».

Séance 6: Travail à partir de la consigne : «La liste de mots montre bien que les arbres ne sont pas silencieux, les mots que vous avez choisis ne veulent peut-être pas tous dire exactement la même chose. Comment pourrait-on les classer ? Dans la liste n'y -a-t-il pas des mots-clés ? Pourraient-ils servir de «mots-titres » ${ }^{10}$

Séance bilan : confrontation des représentations finales des élèves sur le texte avec les représentations initiales. Débat autour de la notion de mots-clés sur l'intégralité de l'ouvrage et évaluation des mots capitalisés, réutilisés, réactivés, à l'oral, à l'écrit, mots que les enfants se sont appropriés de différentes façons.

\section{Premiers commentaires}

L'analyse de ce dispositif revient à souligner les difficultés d'un pari: envisager un compromis entre une observation réfléchie de la langue permettant d'aborder les usages spécifiques du lexique en littérature et un traitement systématique du vocabulaire du texte pour construire le rapport des élèves à la langue. La finalité du travail dans les séances 5 et 6 consiste à résoudre un problème d'ordre lexical (fréquence et récurrence des termes relevant des deux champs repérés : «bruits » et «mouvements ») pour engager la compréhension et l'interprétation du texte. La démarche privilégie des moments méta-lexicaux qui fondent l'entrée dans la littérature par la maîtrise de la langue. Mais cela s'obtient au prix d'une certaine raideur didactique. L'hypothèse est que le recensement et la mise en discussion de

\footnotetext{
${ }^{10}$ Ici intervient la variante proposée dans la classe d'A. Berrini : « Après le relevé des mots, des expressions, des phrases concernant le bruit et le mouvement, aujourd'hui nous allons tenter de les mettre en scène. J'ai choisi certains passages, je les ai écrits sur des petits papiers et vous allez jouer, mimer ce qui est écrit.».
} 
2008 : «Les enjeux lexicaux de la lecture littéraire », dans F. Grossmann et S.Plane (éds), Les Apprentissages lexicaux : Lexique et production verbale, Villeneuve d'Ascq : Presses du Septentrion. Actes des journées INRP/Lidilem, «Lexique et production verbale : vers une meilleure intégration des apprentissages lexicaux », mars 2005, INRP, Lyon

ces deux champs lexicaux vont permettre aux élèves dans un premier temps d'appréhender les difficultés de transcription de réalités visuelles et auditives simples («des feuilles qui se mettent à trembler») ou complexes («les peupliers bâillaient moins bruyamment, en faisant de petites respirations aiguës »), dans un second, d'en apprécier la signification.

Cependant, il n'est pas sûr que relevés et classements soient le moyen adéquat pour y parvenir : on constate en effet que les mots repérés sont nombreux, mais les élèves éprouvent des difficultés à distinguer ceux qui sont porteurs d'un enjeu réel pour la compréhension et l'interprétation du texte et ceux qui, bien que présents dans le texte, ne constituent pas un ancrage pertinent pour en rendre compte. De plus des difficultés de compréhension persistent que ni le contexte ni le travail en langue ne parviennent à lever. Et les séances de mime dans la classe d'Annick Berrini, si elles modifient sensiblement la relation au texte, ne règlent pas tout : le travail sur l'énoncé "le petit garçon voyait les arbres desserrer progressivement leur étreinte », pourtant mimé avec bonheur par un groupe d'élèves, n'a pas permis à la classe de trouver une définition pertinente du mot «étreinte ». Outre la difficulté qu'il y a à préciser les sèmes du mot (saisir un corps en le serrant fortement), l'emploi métaphorique (les arbres assimilés à des corps crispés) et le processus de personnification s'ils ne pénalisent pas l'interprétation ne favorisent pas le travail de définition.

En outre, les exemples de relevés et de classements effectués au cours de la séance 5 («rester au même endroit / dormir / être mort / sommeil épais / léger») montrent que le travail extensif (rendre compte de tous les mots, expressions, phrases qui traduisent le mouvement et le bruit que font les arbres) amène la classe à envisager un travail intensif (établir des gradations dans chacun des deux champs considérés, mais surtout évaluer les énoncés où un même terme peut à la fois désigner un bruit et un mouvement, comme «craquer »).

De fait, la plupart des mots et expressions retenus par les élèves font appel à des images qui associent le bruit et le mouvement, s'inscrivent dans des métaphores qui autorisent des glissements perceptifs autant que sémantiques (ex : «les branches desserrent leur étreinte »).

Mais c'est là, paradoxalement, que le clivage entre travail en langue et en contexte nous semble pouvoir être dépassé, car l'opération de recension, si lourde soit-elle, a fait émerger après coup un aspect important du traitement lexical dans le récit, le jeu subtil de modalisation entre être et paraître qui conditionne l'adhésion au projet fictionnel conçu par Le Clézio («les arbres ont l'air immobile ... »: pour qui ? Pourquoi ?). Une modalisation énonciative non pointée au moment de l'élaboration des séances par les enseignants et qui ne l'est pas davantage par les élèves. En outre, dans la classe de Florence Ozil (séance 6) aux mots «attendus » (considérés comme importants pendant la préparation) se sont ajoutés les mots «trouvés » par les élèves ou ceux qui ont occasionné une négociation plus importante (quantitativement et qualitativement) que prévu: «sifflements sourds», «respirations aiguës »).

Un tel « imprévu didactique » donne à penser que les séances autour du lexique dans le cadre de la lecture littéraire autorisent le franchissement de paliers sémantiques et réflexifs.

Dans les trois classes, l'intérêt porté au lexique du bruit a suscité des interrogations portant sur la différence entre son, bruit, voix. Ont été confrontées des représentations et des conceptions du langage, certes programmées par le texte, mais renforcées par le dispositif didactique et les interactions. Lors de la mise en commun du travail de groupe sur le silence apparent des arbres (Florence Ozil, séance 5), un élève explique que le mot "langage », présent dans le texte, a fait l'objet d'une discussion dans son groupe : il a été finalement retenu, mais assorti d'un point d'interrogation pour être discuté avec l'ensemble de la classe. Après le passage des différents rapporteurs, l'enseignante revient sur ce mot. La discussion qui s'engage conduit à une relecture du texte et à l'explicitation d'un passage décisif : les hommes croient que les arbres sifflent uniquement parce que des oiseaux vivent, et donc 
2008 : «Les enjeux lexicaux de la lecture littéraire », dans F. Grossmann et S.Plane (éds), Les Apprentissages lexicaux : Lexique et production verbale, Villeneuve d'Ascq : Presses du Septentrion. Actes des journées INRP/Lidilem, «Lexique et production verbale : vers une meilleure intégration des apprentissages lexicaux », mars 2005, INRP, Lyon

sifflent dans les arbres, mais pour qui sait écouter ce sont les arbres qui parlent, sifflent, respirent. Le mot «langage » est le pivot du passage : à partir de ce terme générique, deux directions lexicales sont possibles : celle autour des mots « voix », « respiration », « aiguës », «sourd »; celle autour des mots «parler», « disent», « répondre ». Le verbe « siffler» est à l'intersection entre les deux pistes qui posent, par-delà le texte, le problème de la distinction entre la parole comme voix (expression) et la parole comme outil de communication. Ce qui donne l'occasion de relancer l'interprétation du texte et de penser un autre rapport au monde et à la nature.

\section{Conclusion}

Un enseignement du lexique intégré aux séances de lecture littéraire consisterait par conséquent, non pas à jouer de la relation travail en langue / en contexte, mais à évaluer dans l'amont de la rencontre du texte avec la classe, pendant les échanges et dans les travaux les mots qui :

- doivent être identifiés (base minimale de compréhension et d' «accroche» lexicale)

- doivent / peuvent être négociés

- doivent / peuvent être délaissés

- doivent /peuvent être identifiés par les élèves comme « saillants »

De fait, le travail sur le lexique du mouvement se trouve au point de convergence entre différents possibles : ceux de l'auteur, du texte, des mots eux-mêmes, du maître, de chaque élève, de la classe. Les définitions, mêmes approximatives, d'un mot sont indispensables pour que la classe parvienne au partage des significations, mais la complexité de la tâche tient peutêtre au fait que ces moments métalexicaux où se joue l'écart entre langue et discours ne sont pas suffisamment situés par rapport aux intentionnalités : c'est une chose de relever des termes qui disent les mouvements que font les arbres ou «les mots qui plaisent» dans $L e$ Miroir déformant, d'en préciser les sens, les composantes morphologiques, cela en est une autre d'effectuer ce même travail pour dire si l'on accepte ou non l'idée que les arbres parlent, que le miroir a des propriétés magiques ou s'il ne s'agit, dans l'univers de Le Clézio, que d'un monde imaginé par un enfant qui s'ennuie.

On entrevoit alors la possibilité de reconsidérer le développement des compétences lexicales sous l'angle d'ajustements progressifs indispensables entre la prise en compte du lexique du texte (donner à entendre et voir les arbres autrement), les objectifs du maître (stabiliser le sens des mots du texte pour remonter jusqu'à l'intentionnalité littéraire), et l'appropriation qu'en fait la classe (s'emparer du lexique pour échanger). 
2008 : «Les enjeux lexicaux de la lecture littéraire », dans F. Grossmann et S.Plane (éds), Les Apprentissages lexicaux : Lexique et production verbale, Villeneuve d'Ascq : Presses du Septentrion. Actes des journées INRP/Lidilem, «Lexique et production verbale : vers une meilleure intégration des apprentissages lexicaux », mars 2005, INRP, Lyon 